\title{
A Research on Collaborative Innovation Platform for University Environmental Big Data
}

\author{
$\mathrm{Li} \mathrm{ZHOU}^{1, \mathrm{a}^{*}}$ \\ ${ }^{1}$ School of Economics and Management, Zhaoqing University, Zhaoqing, Guangdong Province, \\ China \\ a*13322977@163.com
}

\begin{abstract}
Keywords: Environmental Protection, Big Data, New Research and Development Platform, Collaborative Innovation.
\end{abstract}

\begin{abstract}
The collaborative innovation platform for environmental big data assembles R\&D capabilities from many fields of expertise such as computer science and technology, IOT engineering, network engineering and software engineering, together with professional techniques like environmental protection computer application and big data mining, which is the important supporting power of environmental protection industry development in the intelligent era. University can construct a scientific research innovation team of big data by building a collaborative innovation platform for environmental big data so as to promote the combination of environmental protection business and platforms such as industrial Internet and big data application technology and to make discipline and talent developments better work for the environmental protection business.
\end{abstract}

\section{Introduction}

In recent years, with the development of economic society and industrialization, problems such as environmental quality decline have become the focus of society, the short-board that our country urgently needs to make up through improving the environment. Improvement of technological architecture design of collaborative innovation platform for environmental big data and further exploration and application of environmental big data provide information users at all level with the advanced and suitable analysis management tools and bring new reform to environmental policy-making and governance mechanism. In addition, our country is in an urgent need of expert professionals who specialize in environmental pollution control and protection technology as a result of the lack of high-end talents in environmental protection industry such as excellent entrepreneurs and technical experts. With the help of the construction of collaborative innovation platform for environmental big data, university can improve teaching environment, make greater efforts for professional and technical personnel training of computer application, enlarge area of research, increase research capacity, cultivate expert professionals, incubate entrepreneurs and provide powerful support for the subject cluster like computer science and technology, IOT engineering, network engineering and software engineering, in order to meet the requirement of subject cluster' $\mathrm{s}$ trans-disciplinary, multi-disciplinary, pluralistic construction and development, and to offer better service to environmental big data R\&D and environmental protection industry.

\section{Literature Review}

Zhang Hongjian and $\mathrm{Wu}$ Yong find that data center software platform can solve the problems about non-efficient use and lack of consistency of data; mobile environmental protection public application can enhance public participation in environmental protection; environmental public opinion analysis platform can improve the analysis and tracking of Environmental Protection Agency in terms of environmental public opinion warning.

$\mathrm{Gu}$ Weiwei finds that big data science, as a new multi-field interdisciplinary subject, has gradually formed. Applying the thought and method of big data can unify the environmental data monitor, 
collection, communion and analysis, which can effectively enlarge the width, depth, and reliability of environmental data collection. Meanwhile, it can absorb the merits of thought and model method of different subject fields and then produce greater value.

Luo Junjie and Li Zhixin put forward that big data provides strong technical support for environmental public good promotion and a roadmap is required to make a strategy plan in the construction of the mechanism of development and utilization of environmental big data: a comprehensive survey about supply-demand condition of environmental public goods to obtain complete basic data; establishment of goal and task of the development and utilization mechanism of environmental big data; the design of data sensing, processing, transmission and application system suitable for the environmental big data operating characteristics; the construction of data processing platform fitting the flow law of big data.

Li Guojie proposes that the escalating of environmental data offers wider space to environmental informatization, expand the environmental data application range and provide unlimited potential for the innovation of environmental management model. Big data comprehensively and continuously records environmental influential process and under the comprehensive supervision by using big data, the more monitoring points are set, the more monitoring data can be obtained. The increase of the density of monitoring site can realize a full range, full coverage, full time, all-weather and whole-process environmental regulation.

$\mathrm{Li} \mathrm{Na}$, Tian Yingiie and Shi Yong point out that with the development of big data science, the thought and method of the big data have provided a lot of help for the research on the environmental protection, have improved the reliability of the result, and have brought new revolution for environmental policy formulation and management mode in the meantime. For researchers, pollutant data can be corresponded to economic data from all departments in general balanced economic mode framework, by means of constructing different environmental policy cases for simulation analysis, such as reducing the consumption of fossil energy, adjusting the industrial structure, improving the oil quality, developing new energy vehicles, levying environment taxes and so on. Big environmental data can also effectively guide the public environmental behavior.

\section{Framework for Design}

\section{Goal}

With the help of environmental industrial park, universities can build environmental automatic monitoring platform, geographical information platform, mobile application platform, environment public application platform, and e-government platform. From the aspects of environmental supervision business like environmental law enforcement, environmental impact assessment, environmental emergency, and ecological environment monitor, strengthen the multidimensional supervision of pollution sources in key industries, key areas and key enterprises, gradually realize timely finding, warning, solution, and enhance the work efficiency of environmental supervision.

\section{Framework}

\section{Data Sensing System.}

Through technologies such as the IOT (IOT), intelligent sensing and cloud computing, sensing system of environmental monitoring data including emission of pollution sources, environmental quality can be constructed, and data fusion and real-time transmission and application of all kinds of monitoring equipment like pollution discharge, fixed-point sampling of environmental quality, automatic monitoring, live video and mobile terminal can also be realized.

\section{Data Management System.}

By big data analysis and the IOT technology, with water, gas, noise, vegetation, soil and other ecological elements as evaluation factors, build a system which is able to collect, deliver, store, 
process, maintain and use environmental information from nature reserve and major drainage basins, construct a dynamic evaluation system of ecological environment and its corresponding information management system, and support the operation, management and decision making of environmental protection organization.

\section{Data Storage System.}

Provide big data processing and computing service for data (monitoring) collection, storage and mining; use mobile cloud development platform to provide structure design for environmental big data when processing cloud storage and cloud database at the PaaS level; impel the application of key technologies of cloud computing platform management: the development of cloud service components like cloud messaging, virtual machine and App engine.

\section{Data Display System.}

Construct data distribution center, report processing center and decision-making assist and support center which can become the hub of information resource management system. Construct supervision and management system of motor vehicle exhaust test so as to realize the data exchange between motor vehicle exhaust test basic data, environmental mark and testing organization managements, business query and statistical analysis, gross nitrogen oxides emission reduction analysis, assessment, vehicle emission analysis, and departments including EPA, public security traffic management department, and transportation department. Collect the data scattered in various modular business processing systems to provide data extraction and query service for organizations at all levels; develop and manage information platform system to realize the comprehensive query and analysis, the sharing of statistical information such as comprehensive statistical report, regulatory report and business report and to establish linkage query statistics.

\section{Data Application System.}

In the field of social application, to further improve the judicial efficiency of environmental scene and standardize the law enforcement process, establish a mobile law enforcement information system for environmental watchdog by mobile Internet technology, mobile Internet terminals and environmental big data center. Through the research on resource and environment information system, build a basic database of environmental impact assessment, an environmental pollution survey database and integrated application system.

In the field of teaching application, build a collaborative innovation platform for big data research institute relying on university thus to provide local environmental protection industry services with the environmental protection service platform construction and deployment and infrastructure environment for operation and maintenance. The use of database resource enhances teaching environment, broadens research field, improves the research level, and builds high-level collaborative innovation and R\&D platforms of big data, meeting requirements of high starting point and high technology.

Through the construction of "key platform for environmental IOT", "satellite remote sensing platform for water quality monitoring", "key platform for cloud computing service", "parallel / distributed computing platform" and "university environmental big data storage center", collaborative innovation platform for university environmental big data, on the one hand, strongly supports the development of university research, application service, discipline and professional construction. On the other hand, it provides local environmental protection industry with the environmental protection service platform construction and deployment and infrastructure environment for operation and maintenance; exactly defines the content and direction for environmental protection industry. 


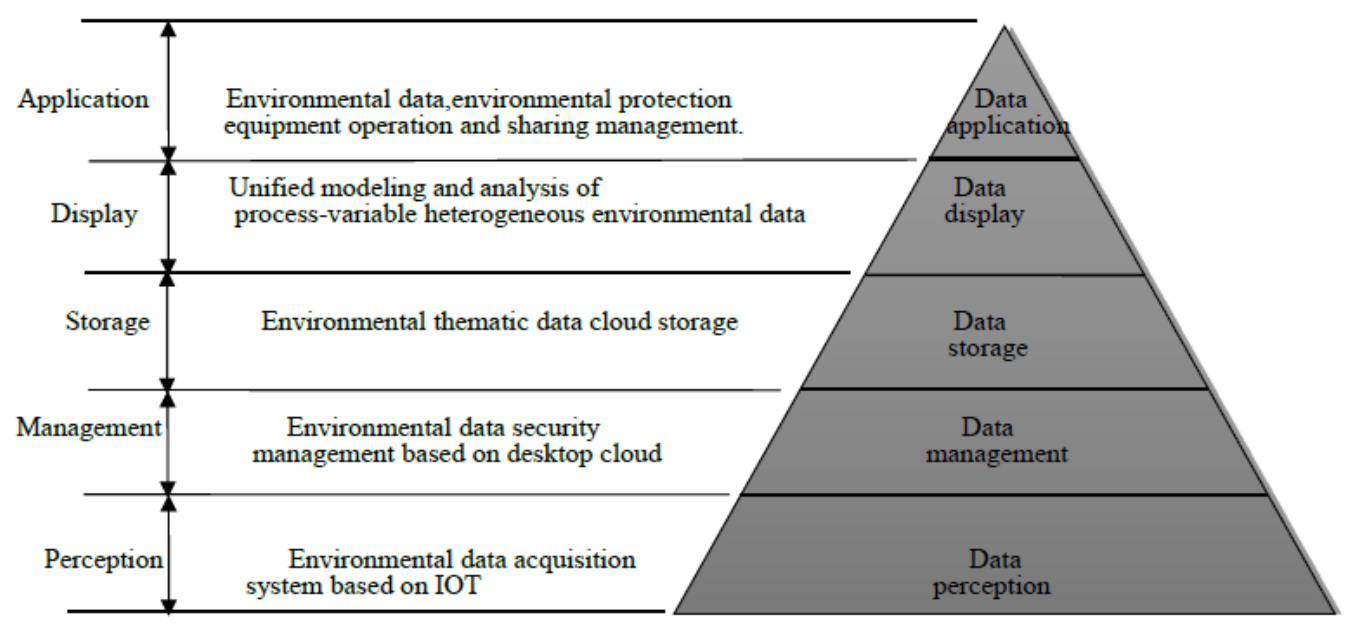

Fig. 1. Core Function Structure of Collaborative Innovation Platform for Environmental Big Data

\section{Core Content}

\section{Collaborative Innovation Platform for Environmental Big Data}

Use new technologies like the IOT and mobile Internet so as to broaden the channel of data collection and to build a cloud service platform based on the system framework of environmental monitoring data collection of sensor devices, on the storage database of all kinds of environmental protection data and on the environmental protection data collection of the IOT. The innovation of data collection methods improves full sensing and real-time monitoring capabilities for various environmental elements such as atmosphere, water, soil, ecology, nucleus and radiation and all kinds of pollution sources. To set up a system of ecological environment information resource catalog and to use the management system of information resource catalog system lead to the centralized integration and dynamic upgrade of data resource in the system and the construction of national ecological basic databases like ecological environmental quality, environmental pollution, natural ecology, nucleus and radiation.

\section{Collaborative Monitoring Platform for Satellite Remote Sensing}

The construction of satellite remote sensing monitoring system provides first-hand information resources for ecological environment change and analyzes the influential factors of ecological environment with the change of seasons, industrial and agricultural development condition and industrial distribution. Researches on water quality, atmosphere, soil, forest vegetation and environmental information involving sensor technology, information and communication technology, remote sensing technology can provide the original data for environmental big data.

\section{Resource Sharing Platform for Ecological Environment Data}

To provide various and flexible data retrieval services, helps to form a data sharing mechanism which focuses on direct access to the platform, supplemented by the exchange between departments and the R\&D of ecological environment data product can improve the management and service level of data sharing.

\section{Collaborative Innovation Platform for Environmental Protection IOT}

By using wireless sensor network technology and mobile Internet technology, build a collaborative innovation platform for water and atmospheric pollution monitoring in the service of environmental data collection and carry out researches based on data analysis model, correlation model and service prediction model of environmental protection and the research on $R \& D$ of environmental monitoring 
system, design and development of environmental cloud services platform and environmental big data application.

\section{Continuous Deployment and Delivery Platform for Environmental Protection Service}

Based on new technologies such as IOT and mobile Internet and other, establish system integration services with real-time data monitoring, early warning, decision making and application based on cloud computing platform. Based on geographic information system, build a multi-tier resource and environment information model. According to data collection system of resource and environment information and the predictive and simulate model of environmental event's impact, set up a continuous integration production platform used for the development of the environmental protection service platform. Based on micro service component of environmental protection and environmental protection service cloud computing platform, the government and the enterprises are able to obtain data to access API.

Table 1. Core Content of Collaborative Innovation Platform for University Environmental Big Data

\begin{tabular}{|l|l|}
\hline \multicolumn{1}{|c|}{ Platform } & \multicolumn{1}{|c|}{ Core Content } \\
\hline $\begin{array}{l}\text { Collaborative Service Platform for } \\
\text { Environmental Big Data }\end{array}$ & Cloud Service of Environmental Protection Data Collection based on IOT \\
\hline $\begin{array}{l}\text { Envilaborative Service Platform for } \\
\text { Environmental Big Data }\end{array}$ & Cloud Service of Environmental Protection Data Collection based on IOT \\
\hline $\begin{array}{l}\text { Collaborative Monitoring Platform for } \\
\text { Satellite Remote Sensing }\end{array}$ & $\begin{array}{l}\text { To Provide the Most Original Data for Environmental Big Data by Sensor } \\
\text { Technology, Information and Communication Technology and Remote }\end{array}$ \\
\hline $\begin{array}{l}\text { Resource Sharing Platform for } \\
\text { Ecological Environment Data }\end{array}$ & To Provide Various and Flexible Data Retrieval Services \\
\hline $\begin{array}{l}\text { Collaborative Innovation Platform for } \\
\text { Environmental Protection IOT }\end{array}$ & $\begin{array}{l}\text { Po Build a Collaborative Innovation Platform for Water and Atmospheric } \\
\text { Service Prediction }\end{array}$ \\
\hline $\begin{array}{l}\text { Continuous Deployment and Delivery } \\
\text { Service }\end{array}$ & $\begin{array}{l}\text { To Build a Continuous Integration Production Platform Used for the } \\
\text { Development of Environmental Protection Service Platform based on } \\
\text { Micro Service Component of Environmental Protection and Environmental } \\
\text { Protection Service Cloud Computing Platform }\end{array}$ \\
\hline
\end{tabular}

\section{Conclusion}

With the perfection of environmental big data construction, collaborative innovation platform for university environmental big data realizes the data collection, cleaning, analysis and decision-making support through IOT, cloud computing and big data technology and besides, it is qualified for real-time monitoring of environment governance effect and the dynamic update of countermeasure. Its strategic significance is embodied in the professional process of environmental data which can be transformed into information with decision-making function, so as to improve the comprehensive decision-making capability and public service ability of the environmental protection department. Meanwhile, with the open data, practical data and cases of environmental governance can be spread to the public, which is a way to encourage the participation of the whole society in order to improve the effect and efficiency of environmental protection. Also, the collaborative innovation platform for university environmental big data shows incomparable advantages in the cultivation of practical 
talents of environmental protection. From the professional study, the students can get involved in environmental project management and accumulate career experience in advance.

\section{References}

[1] W. Los, J. Wood, Dealing with Data: Upgrading Infrastructure, Science. 331 (2011) 639-806.

[2] D. Agrawal, S. Das, A. El Abbadi, Big Data and Cloud Computing: Current State and Future Opportunities, International Conference on Edbt. 65 (2011) 530-533.

[3] Gu Weiwei, How Big Data Help Protect the Environment? China Environment News, 2014.

[4] J. Langford, Parallel Machine Learning on Big Data, XRDS: Crossroads, The ACM Magazine for Students. 19 (2012) 60-62.

[5] Luo Junjie, Li Zhixin, On the Development and Use Mechanism of Environmental Big Data Based on Road Map, Journal of Central South University of Forestry and Technology(Social Sciences). 3 (2016) 08-11.

[6] Li Guojie, Scientific Value of Big Data Research, Communications of the China Computer Federation. 9 (2012) 07-15.

[7] Li Na, Tian Yingjie, Shi Yong, Big Data Application in Environmental Management, Environmental Protection. 19 (2015) 30-33.

[8] Shi Yong, Big Data and New Technological Challenge, Science and Technology for Development. 01 (2014) 25-30.

[9] Wu Yong, Zhang Hongjian, Smart Environmental Protection Solution Based On Big Data and Cloud Computing, Information Technology and Standardization. 11 (2013) 38-41.

[10] A. Jacobs, The Pathologies of Big Data, Communications of the ACM. 52 (2009) 36-44. 